\title{
MRI AND CT IMAGE FUSION BASED STRUCTURE-PRESERVING FILTER
}

\author{
Qiaoqiao $\mathrm{Li}^{1}$, Guoyue Chen ${ }^{2}$, Xingguo Zhang ${ }^{2}$, Kazuki Saruta ${ }^{2}$ and \\ Yuki Terata ${ }^{2}$ \\ ${ }^{1}$ Graduate School of Systems Science and Technology, \\ Akita Prefectural University, Akita, Japan \\ ${ }^{2}$ Information and Computer Science, \\ Akita Prefectural University, Akita, Japan
}

\begin{abstract}
Medical image fusion plays an important role in clinical application such as image-guided radiotherapy and surgery, and treatment planning. The main purpose of the medical image fusion is to fuse different multi-modal images, such as MRI and CT, into a single image. In this paper, a novel fusion method is proposed based on a fast structure-preserving filter for medical image MRI and CT of a brain. The fast structure preserving filter is a novel double weighted average image filter (SGF) which enables to smooth out high-contrast detail and textures while preserving major image structures very well. The workflow of the proposed method is as follows: first, the detail layers of two source images are obtained by using the structurepreserving filter. Second, compute the weights of each source image by calculating from the detail layer with the help of image statistics. Finally, fuse source images by weighted average using the computed weights. Experimental results show that the proposed method is superior to the existing medical image fusion method in terms of subjective evaluation and objective evaluation.
\end{abstract}

\section{KEYWORDS}

Multimodal image fusion, structure-preserving filter, weighted average.

\section{INTRODUCTION}

With the development of the computer science, there are many modalities of medical images to support more accurate clinical information to physicians for better medical analysis and diagnosis. Today many kinds of modalities of medical images are existing, such as computed tomography (CT), magnetic resonance angiography (MRA), magnetic resonance imaging (MRI), positron emission tomography (PET) and single photon emission tomography (SPECT) [1-3]. Different modality medical images can provide different perspectives on the human body, such as CT image can provide sense structures like bones and implants with less distortion, while the MRI image can provide normal and pathological soft tissue information [1-5]. Therefore, in order to fully diagnose the condition of patients, it is desired to fusing different modality medical images into a single image, called image fusion, such that all the information is available.

Image fusion can be divided into three levels: pixel levels, feature levels and decision levels [5-6]. Due to the advantage of pixel level method, such as containing the original measured quantities, easy implementation and computationally efficient, we focus the pixel level method in this paper.

Natarajan Meghanathan et al. (Eds) : SPPR, SCAI, CSIA, WiMoA, ICCSEA, InWeS, NECO, GridCom - 2018 pp. 127-135, 2018. (C) CS \& IT-CSCP 2018

DOI : $10.5121 /$ csit.2018.81712 
Pixel-level image fusion method can be divided into two categories: spatial domain algorithms and transform domain algorithms [8]. In the spatial domain, Calhoun et.al use the technology of independent component analysis (ICA) for their fusion method [9]. Patil et.al introduce the principal component analysis (PCA) technology in their fusion method [10]. Recently, structurepreserving smoothing filter technology is applied in the fusion method. For example, Zhan et.al [11] apply a fast filter to accelerate their fusion method. In transform domain method, Alfano et.al [12] and Vekkot [13] propose the fusion method based on wavelet and Das et.al use nonsubsampled contourlet transform (NSCT) in their fusion method [14] and so on. In addition to the fusion methods used wavelet and contourlet transform, many researches also have introduced the structure-preserving smoothing filter into their fusion methods. Such as, Li et.al [15], Bavirisetti et.al [3] and Zhan et.al [16] use the guide filter (GF) to obtain the fusion image, Kumar et.al [6] introduce the cross bilateral filter (CBF) into their fusion scheme.

Considering the edge preserving filter can extract effectively salient information from the source images, a new fast structure-preserving filter [17], a novel double weighted average image filter (SGF) based on the segment graph which is introduced into the modalities medical image fusion in this paper. In [17], Zhang et.al have proved that SGF can keep the major edges better than the GF and CBF. In this paper, a new method is proposed. First, use the SGF smooth the source image. Second, subtract the smooth image from the source image to obtain the detailed information. Third, use a weighted average method to fuse the source image. The weighted average based fusion method has been employed in [36] and the fusion results have shown good performance.

\section{ALGORITHM}

\subsection{DOUBLE WEIGHTED AVERAGE IMAGE FILTER (SGF)}

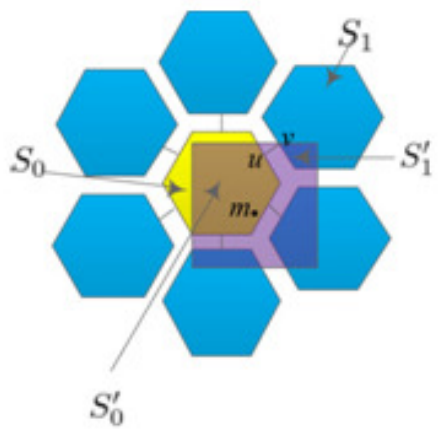

Figure 1. Filter kernel of structure-preserving filter (SGF)

Zhang et.al proposed a novel double weighted average image filter used the segment graph [17]. Because super pixel decomposition of a given image has been studied and the superpixel can run very fast in linear, they use the super pixel decomposition to construct the segment graph. A detailed introduction of the segment graph can be seen from Zhang et.al's literature.

Because the novel structure preserving structure based on the double weight, i.e. internal weight and external weight, we will introduce them in the next. Considering the tree distance which has the edge-aware property, the internal weight function $w_{1}$ can be defined by

$$
w_{1}(m, n)=\exp \left(-\frac{D(m, n)}{\sigma}\right),
$$


where $D(m, n)$ represents the tree distance between pixels $m$ and $n$. As $\sigma$ controls the attenuation speed of $D(m, n)$, the $w_{1}$ is inversely proportional to the tree distance $D(m, n)$.

In order to describe external weight, a smoothing window $\mathrm{w}_{n}$ is introduced which is shown in Fig. 2 (purple square). As shown in Fig.2, several super pixel regions are denoted as $\left\{S_{0}, S_{1}, \mathrm{~L}, S_{k}\right\}$ and the overlapped regions are represented by $\left\{S_{0}^{j}, S_{1}^{j}, \mathrm{~L}, S_{k}^{j}\right\}$, namely $S_{i}^{j}=\mathrm{w}_{n} \cap S_{i}$, the external weight function $w_{2}$ can be defined by the area size ration $S_{i}^{j}$ and $S_{i}$ :

$$
w_{2}(m, n)=\frac{\left|S_{i}^{j}\right|}{\left|S_{i}\right|} \text {. }
$$

Once the double weights are obtained, the filter output of an input image $I$ at pixel $n$ can be given by

$$
J_{n}=\frac{1}{K_{n}} \sum_{0 \leq i \leq k} w_{2}\left(n, S_{i}\right) \sum_{m \in S_{i}} w_{1}(n, m) I_{m}
$$

where $K_{n}, S_{i}$ and $J_{n}$ represent a normalizing term, super pixel region and filter output. $w_{1}$ and $w_{2}$ are the internal weight function and external weight function, respectively. The output $J_{n}$ at the pixel $n$ is the double weighted average of the intensity value $I_{m}$ in a specific neighbour region $\Omega=\mathrm{U}_{0 \leq i \leq k} S_{i}\left(m \in S_{0}\right)$.

\subsection{FOCUS RULE}

In this paper, we adopt a weighted average method to fuse the images. The weighted average fusion rule is proposed by Shah et.al [18] and Kumar has used this fusion rule for their method [6].

Shah et.al compute the weight of wavelet coefficient [18], instead of it we compute the weight of the detail coefficient. The weight is computed in a window of size $w \times w$ around a detail coefficient $A_{d}(i, j)$ or $B_{d}(i, j)$ which is denoted as a matrix $R$. Let us treat each row of $R$ as an observation and column as a variable, and then unbiased estimate $C_{h}^{x, y}$ of its covariance matrix [19] can be computed by

$$
\begin{aligned}
\operatorname{cov} \operatorname{ariance}(X) & =E\left((R-E(R))(R-E(R))^{T}\right) \\
C_{h}^{x, y} & =\frac{\sum_{i=1}^{n}\left(r_{i}-\bar{r}\right)\left(r_{i}-\bar{r}\right)^{T}}{n-1}
\end{aligned}
$$

where $r_{i}$ is the $i$-th observation of the $n$-dimensional variables and $\bar{r}_{i}$ is the mean of observations. It can be observed that diagonal of $C_{h}^{x, y}$ is a variance vector of each column of the matrix $R$. Then compute eigenvalues of $C_{h}^{x, y}$, denoted by $\lambda_{H}^{j}$, and the number of eigenvalues depend on the size of it. Since the size of $C_{h}^{x, y}$ is $w \times w$, the number of Eigen values is $w$. The sum of these eigenvalues is directly proportional to the strength of horizontal edges and the sum can be named by edgestrength $h_{h}$ 


$$
\text { edgestrength }(\mathrm{x}, \mathrm{y})=\sum_{j=1}^{n} \lambda_{H}^{j} .
$$

Take consideration of the vertical edges, an unbiased covariance estimate $C_{v}^{x, y}$ is computed under the condition of treating each column $R$ as an observation and row as a variable, and then vertical edge strength can also obtained by summing the eigenvalues $\lambda_{V}^{j}$ of it

$$
\text { edgestrength }(x ; y)=\sum_{j=1}^{n} \lambda_{v}^{j}
$$

For a particular detail coefficient at the location $(x, y)$, the weight is obtained by adding the horizontal edge strength and vertical edge strength

$$
w d(x, y)=\text { edgestrength }_{h}(x, y)+\text { edgestrength }_{v}(x, y) .
$$

Considering the Eq.2.6 and Eq.2.7, the weight can be rewritten by

$$
w d(x, y)=\sum_{j=1}^{n} \lambda_{H}^{j}+\sum_{j=1}^{n} \lambda_{V}^{j} .
$$

Then, the fused image can be obtained by

$$
F(x, y)=\frac{I_{1}(x, y) * w d_{1}(x, y)+I_{2}(x, y) * w d_{2}(x, y)}{w d_{1}(x, y)+w d_{2}(x, y)} .
$$

\section{PROPOSED MULTI-FOCUS FUSION SCHEME}

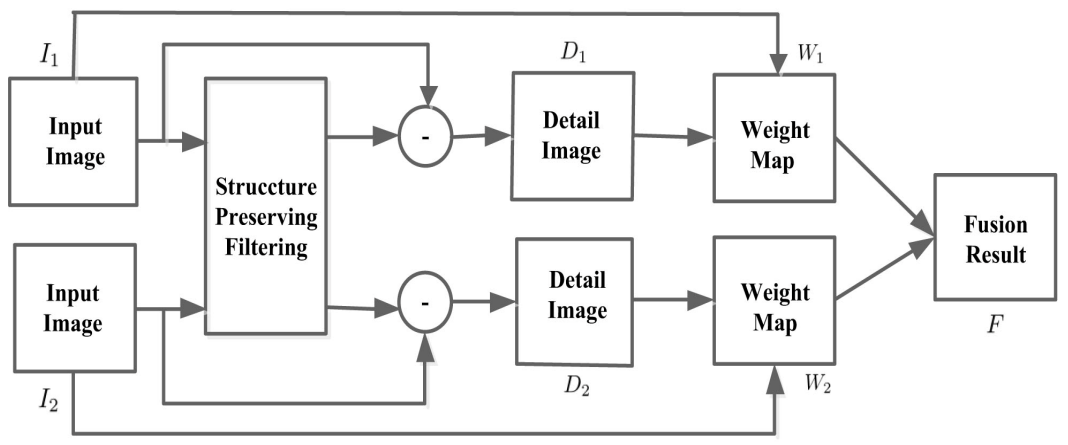

Figure 2. System diagram of the proposed fusion framework.

In this section, the proposed fusion method will be introduced in detail. Figure 2 shows the framework of the proposed fusion method. For two perfectly registered source images denoted by $I_{1}$ and $I_{2}$, the proposed algorithm consists of three main steps as shown in Figure 2.

Step 1: The two source images are first decomposed into approximation images ( $A_{1}$ and $A_{2}$ ) and derail images ( $D_{1}$ and $D_{2}$ ) by structure-preserving filter 


$$
A_{k}=S G F\left(I_{k}\right), \quad k=1,2 .
$$

And then, the detail images are computed by subtracting the approximation images from source images

$$
D_{k}=I_{k}-A_{k}, \quad k=1,2 \text {. }
$$

Step 2: Use the detail images to computed the weight map of each image at the location (x, y)

$$
W_{k}(x, y)=\sum_{j=1}^{n} l_{H}^{j}+\sum_{j=1}^{n} l_{V}^{j}, \quad k=1,2 .
$$

Step 3: Once the weight map is obtained, the fusion image can be computed as followings

$$
F(x, y)=\frac{I_{1}(x, y) * W_{1}(x, y)+I_{2}(x, y) * W_{2}(x, y)}{W_{1}(x, y)+W_{2}(x, y)}
$$

\section{EXPERIMENT RESULTS}

\subsection{FUSION EVALUATION METRICS}

In order to evaluate the fusion result performance of the proposed method, two objective image fusion performance metrics are adopted to evaluate performances of different fusion, i.e. structure-based metric $Q_{w}^{x y l f}$ [20] and normalized mutual information $Q_{M I}$ [21].

\subsubsection{STRUCTURAL SIMILARITY-BASED METRIC $\left(Q_{w}^{x y l f}\right)$}

The structural similarity (SSIM) metric measures the corresponding regions in a reference source image $A$ or $B$ and the fusion image $\mathrm{F}$ with a sliding window $\mathbf{w}$ which can be defined by

$$
\operatorname{SSIM}(A, F \mid w)=\frac{\left(2 \bar{w}_{A} \bar{w}_{F}+C_{1}\right)\left(2 \delta_{w_{A}} \delta_{w_{F}}+C_{2}\right)\left(\delta_{w_{A} w_{F}}+C_{3}\right)}{\left(w_{A}^{2}+w_{F}^{2}+C_{1}\right)\left(2 \delta_{w_{A}} \delta_{w_{F}}+C_{2}\right)},
$$

the detailed parameter settings of it can be seen from [22] [23]. Yang et.al [20] proposed a new metric based on SSIM which can be written by

$$
Q_{w}^{a b \mid f}= \begin{cases}\lambda_{w} \operatorname{SSIM}(A, F \mid w)+\left(1-\lambda_{w}\right) \operatorname{SSIM}(B, F \mid w), & \operatorname{SSIM}(A, B \mid w) \geq 0.75 \\ \max (\operatorname{SSIM}(A, F \mid w), \operatorname{SSIM}(B, F \mid w)), & \operatorname{SSIM}(A, B \mid w)<0.75\end{cases}
$$

where the weight $\lambda_{w}$ is defined by

$$
\lambda_{w}=\frac{s(\mathrm{~A} \mid w)}{s(\mathrm{~A} \mid w)+s(\mathrm{~B} \mid w)} .
$$

In implementation, $s(\mathrm{~A} \mid w)$ and $s(\mathrm{~B} \mid w)$ are the variance of images $\mathrm{A}$ and $\mathrm{B}$ with the window $\mathbf{w}$. 


\subsubsection{NORMALIZED MUTUAL INFORMATION $\left(Q_{M I}\right)$}

Mutual information (MI) is a quantitative measure of the mutual dependence of two variables. And the mutual information for two discrete random variables $\mathrm{U}$ and $\mathrm{V}$ is defined by

$$
M I(U, V)=\sum_{u \in U} \sum_{v \in V} p(u, v) \log \frac{p(u, v)}{p(u) p(v)},
$$

where $p(u, v)$ is the joint probability distribution function of $\mathrm{U}$ and $\mathrm{V}$, and $p(u)$ and $p(v)$ represent the marginal probability distribution function of $\mathrm{U}$ and $\mathrm{V}$, respectively. Based on the above definition, the normalized mutual information of the fusion image regarding to the source image $A$ and $B$ is computed as

$$
Q_{M I}=2\left[\frac{M I(A, F)}{H(A)+H(F)}+\frac{M I(B, F)}{H(B)+H(F)}\right],
$$

where the $H(A), H(B)$ and $H(F)$ are the marginal entropy of images $A, B$ and fusion image $F$.

\subsection{EXPERIMENT RESULT}

Experiments are carried out on two pairs of CT and MRI modality medical images as shown in Figure 3 (a), (b) and Figure 4 (a), (b). The fusion image obtained by the proposed method is compared with the method proposed by Bacirisetti et.al [3]. Bacirisetti et.al use the guide filter to get the detail images and then compute the weight of them using the image statistics (GFS). The experiment results are shown in Figure 3 (c) and Figure 4 (c).

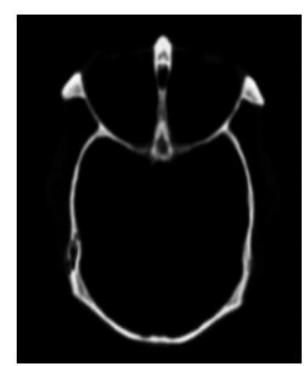

(a)

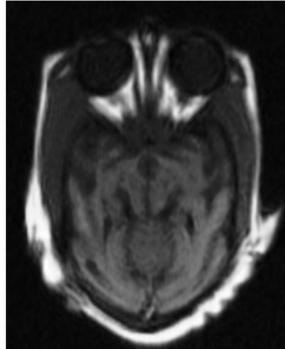

(b)

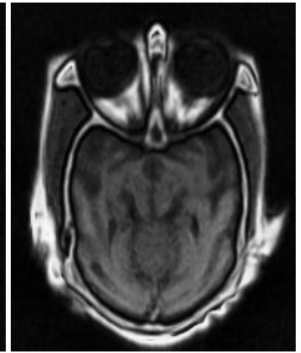

(c)

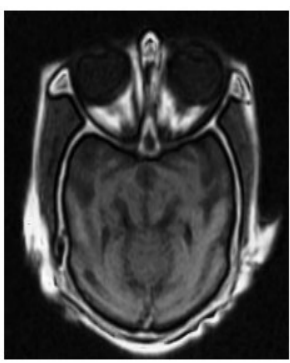

(d)

Figure 3. Fusion result of image A

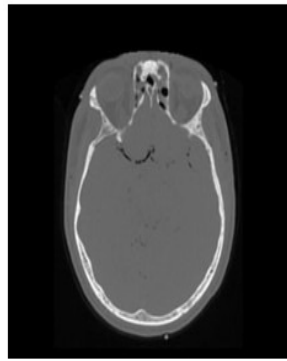

(a)

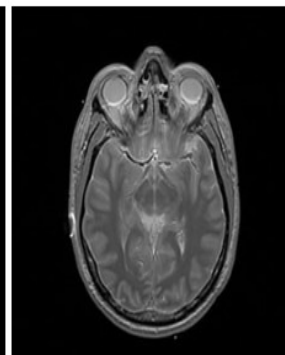

(b)

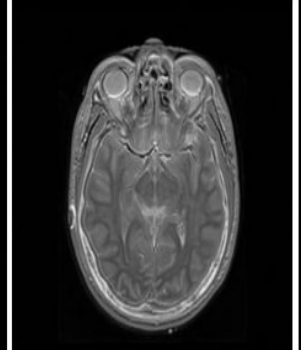

(c)

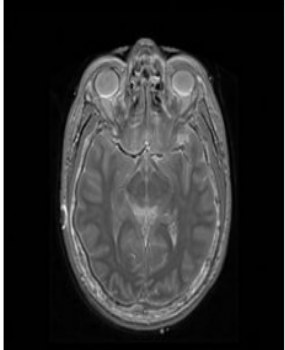

(d)

Figure 4. Fusion result of image B 
Figure 3 shows the fusion results of the test modalities images A. Figure 3 (c) shows the fusion result of GFS. Some "halo" artifacts arise the bright region of the left edge of the fusion result.

Figure 3 (d) shows the fusion result of the proposed method. It can be seen that the proposed method can obtain satisfactory result with keeping more details, such as the fusion result is no "halo" artifacts.

Figure 4 shows the fusion results of the test modality images B. The fusion result of method GFS and the proposed method are shown in Figure 4 (c) and (d), respectively. It can be seen from the Figure 4 (d) that the proposed method's fusion result is not well as the GFS's method in visual quality, but it is not bad.

The objective evaluation of the fused result for the test two pairs images are shown in Table 1. From the Table 1, the fusion result of the proposed method achieves the highest values almost all of the evaluation metrics. Considering the analysis of subjective evaluation and objective evaluation, the proposed algorithm obtains the better fusion result than the GFS method.

Table 1. Objective Performance

\begin{tabular}{|c|c|c|c|}
\hline Image & Metric & GFS & Proposed \\
\hline \multirow{2}{*}{ Image A } & $Q_{w}^{x y \mid f}$ & 0.8516 & 0.9170 \\
\cline { 2 - 4 } & $Q_{M I}$ & 0.7047 & 0.8660 \\
\hline \multirow{2}{*}{ Image B } & $Q_{w}^{x y \mid f}$ & 0.8689 & 0.8868 \\
\cline { 2 - 4 } & $Q_{M I}$ & 0.9277 & 0.9754 \\
\hline
\end{tabular}

\section{CONCLUSION}

In this paper, we have proposed a new fusion method based on structure-preserving filter (SGF). A weighted average method is used as the fusion rule in the proposed method. The weights of the source images are computed from the detail images of them.

In order to demonstrate the effectiveness of the proposed algorithm, two pairs of MRI and CT images have been considered. As shown in the experimental results, the proposed method has obtained better performance than the method in terms of the both visual performance and objective metrics.

\section{ACKNOWLEDGMENTS}

This work has been supported by the China Scholarship Council for Ph.D. program.

\section{REFERENCES}

[1] S. Liu \& T. Zhang \& H. Li \& J. Zhao \& H. Li, (2015) "Medical image fusion based on nuclear norm minimization,” International Journal of Imaging Systems and Technology, Vol.25, No.4, pp310-316.

[2] S. Liu, J. Zhao, and M. Shi, (2015) "Medical image fusion based on improved sum-modifiedlaplacian,” International Journal of Imaging Systems and Technology, Vol.25, No.3, pp206-212.

[3] D. P. Bavirisetti, V. Kollu, X. Gang, and R. Dhuli, (2017) "Fusion of mri and ct images using guided image filter and image statistics," International Journal of Imaging Systems and Technology, Vol.27, No.3, pp227-237. 
[4] A. Wong and W. Bishop, (2008) "Efficient least squares fusion of mri and ct images using a phase congruency model," Pattern Recognition Letters, Vol.29, No.3, pp173-180

[5] G. Bhatnagar, Q. J. Wu, and Z. Liu, (2015) "A new contrast based multimodal medical image fusion frame- work," Neuro computing 157, pp143-152.

[6] B. S. Kumar, (2015) "Image fusion based on pixel significance using cross bilateral filter," Signal, image and video processing, Vol.9, No.5, pp1193-1204.

[7] R. Redondo, F.Sroubek, S. Fischer, and G. Cristobal, (2009) "Multifocus image fusion using the log gabor transform and a multisize windows technique," Information Fusion, Vol.10, No.2, pp163-171.

[8] K. S. Tamilselvan and G. Murugesan, (2014) "Survey and analysis of various image fusion techniques for clinical ct and mri images," International Journal of Imaging Systems and Technology, Vol.24, No.2, pp193-202.

[9] V. D. Calhoun and T. Adali, (2009) "Feature-based fusion of medical imaging data," IEEE Transactions on Information Technology in Biomedicine, Vol.13, No.5, pp711-720.

[10] U. Patil and U. Mudengudi, (2011)“Image fusion using hierarchical pca.," in image Information Processing (ICIIP), 2011 International Conference on, pp1-6, IEEE.

[11] K. Zhan, Y. Xie, H. Wang, and Y. Min, (2017) "Fast filtering image fusion," Journal of Electronic Imaging, Vol.26, No.6, 063004.

[12] B. Alfano, M. Ciampi, and G. De Pietro, (2007) "A wavelet-based algorithm for multimodal medical image fusion," in International Conference on Semantic and Digital Media Technologies, pp117-120, Springer.

[13] S. Vekkot, (2010) "Wavelet based medical image fusion using filter masks," in FIRA RoboWorld Congress, pp298-305, Springer.

[14] S. Das and M. K. Kundu, (2012) "Nsct-based multimodal medical image fusion using pulse-coupled neural network and modified spatial frequency," Medical \& biological engineering \& computing, Vol.50, No.10, pp1105-1114.

[15] S. Li, X. Kang, and J. Hu, (2013) "Image fusion with guided filtering," IEEE Transactions on Image Processing, Vol.22, No.7, pp2864-2875.

[16] K. Zhan, J. Teng, Q. Li, J. Shi, et al., (2015) "A novel explicit multi-focus image fusion method," Journal of Information Hiding and Multimedia Signal Processing, Vol.6, No.3 , pp600-612.

[17] F. Zhang, L. Dai, S. Xiang, and X. Zhang, (2015) "Segment graph based image filtering: fast structure- preserving smoothing," in Proceedings of the IEEE International Conference on Computer Vision, pp361-369.

[18] P. Shah, S. N. Merchant, and U. B. Desai, (2011) "An efficient adaptive fusion scheme for multifocus images in wavelet domain using statistical properties of neighborhood," in Information Fusion (FUSION), 2011 Proceedings of the 14th International Conference on, pp1-7, IEEE.

[19] S. J. Devlin, R. Gnanadesikan, and J. R. Kettenring, (1975) "Robust estimation and outlier detection with correlation coefficients," Biometrika, Vol.62, No.3, pp531-545.

[20] C. Yang, J.-Q. Zhang, X.-R. Wang, and X. Liu, (2008) "A novel similarity based quality metric for image fusion," Information Fusion, Vol.9, No.2, pp156-160. 
[21] M. Hossny, S. Nahavandi, and D. Creighton, (2008) "Comments on information measure for performance of image fusion," Electronics letters, Vol.44, No.18, pp1066-1067.

[22] Z. Wang, A. C. Bovik, H. R. Sheikh, and E. P. Simoncelli, (2004) "Image quality assessment: from error visibility to structural similarity," IEEE transactions on image processing, Vol.13, No.4, pp600612.

[23] Z. Liu, E. Blasch, Z. Xue, J. Zhao, R. Laganiere, and W. Wu , (2012) "Objective assessment of multiresolution image fusion algorithms for context enhancement in night vision: a comparative study," IEEE transactions on pattern analysis and machine intelligence, Vol.34, No.1, pp94-109. 